\title{
A correlation analysis algorithm about index in Power grid planning
}

\author{
LIU Yangjun ${ }^{1, a}$, WANG Qingxin ${ }^{1, b}$, DING Jiaman ${ }^{1, c^{*}}$ \\ ${ }^{1}$ Faculty of Information Engineering and Automation, \\ Kunming University of Science and Technology,Kunming, China \\ ae-mail:723121674@qq.com, be-mail: yunnan403@sina.com, ce-mail: tjom2008@163.com
}

Key words: Lucas correlation model; power grid planning; probability boxes; fusion;

Abstract: Currently,decision scheme often ignores the correlations among the indexes, which will not only make calculation more complicated, but also affect the accuracy of decision-making, even cause huge economic losses. It is unreasonable that it assumed independent irrelevance in information fusion of the indexes in traditional power grid planning. Therefore, the paper bring out the correlation classification schemes and forms, by the way ,put forward the fusion method and Lucas correlation model based on probability theory. The result shows that the method is more timeliness and accurate to solve the problem of correlation and fusion.

\section{Introduction}

With the development of the economy, Urban and rural residents' demand for electricity is growing and power quality becomes increasingly demanding. Affected by uncertainty in the future national policy, economic development, environmental changes, there are also many uncertainties in the network planning process [1].

Currently, in terms of power grid planning at home and abroad,there has been some research, and put forward some methods and models, such as principal component analysis[2], between indicators fusion algorithm[3], the probability boxes theoretical model[4]. In addition, [5] proposed based Hotels troubleshooting grid, all the uncertainty Problem events are included in a closed box, so that the theory can be more comprehensive measure of all the features of the event probability theory cartridge; [6] the basic principles of the Analytic Hierarchy Process to establish a reflection of the macro-control system, power grid construction index scale; literature [7] are based on DS evidence presented information fusion algorithm and weighted data fusion algorithm, the multi-fusion indicators, but none of the early indicators do any treatment.

Therefore, we propose a network planning and correlation of parameters and fusion algorithm based on probability theory box, resulting in a more rational and efficient metrics related fusion algorithms.

\section{Correlation analysis of probability boxes theory}

Probability boxes theory. In nature, the attribute of most things is uncertain. Probability boxes can solve the problem like this, the unknown probability distribution types, source of dependence is unknown, measurement uncertainty, etc.

Figure 1 shows the probability boxes[8].

Lower bound: $\overline{F_{x}}(x)=1-\underline{P}(\mathrm{X}>\mathrm{x})$

Upper bound: $\underline{F}_{x}(x)=1-\underline{P}(\mathrm{X} \leq \mathrm{x})$

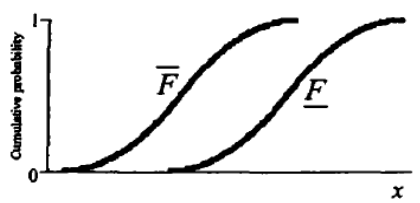

Figure 1: Probability boxes of schematic diagram

Correlation classification strategy based on the theory of the probability boxes. Under normal circumstances, events are not independent, and there is a relevant relationship, If , in the Venn diagram 
depicting events of the relationship between the probability of the event. For the correlation of events A and event $\mathrm{B}$ of the Venn diagram is shown in Figure 2.

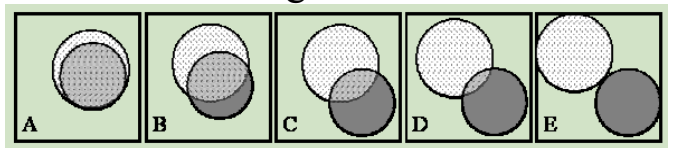

Figure 2: Dependence between two events depicted in Venn diagrams

Picture A denotes positive relevance: small round all the events were included in the great circle;

Picture E denotes the negative relevance: all happened in a small circle of events do not occur in the great circle;

Picture $\mathrm{C}$ denotes the weak relevance: if the probability of $\mathrm{A}$ and $\mathrm{B}$ occurred together is small, or less, that the two events is not related,

Picture B denotes the positively strong relevance: between all the relatation and independence;

Picture D denotes negatively strong correlation, between all negatively relatation and independence;

Use "and" and "or" to show above relevance: (perfect)

$$
P(\mathrm{Al} \mathrm{B})=\operatorname{and}_{\text {perfect }}(\mathrm{a}, \mathrm{b})=\min (\mathrm{a}, \mathrm{b}) \quad P(\mathrm{AU} \mathrm{B})=\operatorname{or}_{\text {perfect }}(\mathrm{a}, \mathrm{b})=\max (\mathrm{a}, \mathrm{b})
$$

(opposite)

$$
P(A \mid B)=\text { and }_{\text {opposite }}(a, b)=\max (a+b-1,0) \quad P(A \cup B)=\text { or opposite }_{\text {it }}(a)=\min (1, a+b)
$$

(positive)

$$
P(A \mid B)=\text { and }_{\text {positive }}(a, b)=[a b, \min (a, b)] \quad P(A \cup B)=o r_{\text {positive }}(a, b)=[1-(1-a)(1-b), \max (a, b)]
$$

(negative)

$$
P(A \mid B)=\text { and }_{\text {rogative }}(a, b)=[\max (a+b-1,0), a b] \quad P(A \cup B)=\alpha r_{\text {rogative }}(a, b)=[1-(1-a)(1-b), \min (1, a+b)]
$$

Correlation expression based on the theory of the probability boxes.Lucas defines the index correlation, correlation coefficients will be applied to the Lucas related model, this leads to the formulation:

$$
\mathrm{P}(\mathrm{Al} \mathrm{B})=\text { and }_{\text {Luas }}(a, b, r)=a b+r \sqrt{a(1-a) b(1-b)}
$$

Because when $r$ take different values, the correlation of Lucas model possible for more than 1 and less than -1 , the Lucas model reasonable is to limit the input correlations to be no smaller than and no larger than, where

$$
\underline{r}=\frac{\max (a+b-1,0)}{\sqrt{a(1-a) b(1-b)}} \quad \bar{r}=\frac{\min (a, b)-a b}{\sqrt{a(1-a) b(1-b)}}
$$

\section{The grid index correlation analysis}

The correlation model.the papar combining with the provincial power grid planning and original data of multiple evaluation indexes, The correlation model reflecting the early indicators of the provincial power grid planning is set up preliminarily.

(1) Qualified rate of the user terminal voltage. A1 denotes user terminal voltage pass rate,

$$
\mathrm{A} 1=\mathrm{a} 1 / \mathrm{b} 1
$$

Where A1 denotes the sum of the voltage qualification rate of each point, the point voltage qualification rate comes from qualified time divided by running time; b1 is the sum of the number of measurement point.

(2) The average load rate of transformer. A2 denotes average load rate of transformer

$$
\mathrm{A} 2=\mathrm{a} 2 /(\mathrm{b} 2 * \mathrm{c} 2)
$$


Where a2 denotes the total supply of single project transformer during the inspection cycle; b2 denotes the total power transmission capacity of single project transformer during the inspection cycle; $\mathrm{c} 2$ for the hours of the operation of the equipment during the time.

(3)Power supply reliability rate. A3 denotes power supply reliability rate,

$$
\text { A } 3=1-a 3 / b 3
$$

Where a3 denotes the average outage time; b3 denotes statistics of the total time.

The expectation and variance of every is calculated from the above formula using the matlab: $\mathrm{E}(\mathrm{X})=0.9813, \mathrm{~V}(\mathrm{X})=0.0415, \mathrm{E}(\mathrm{Y})=0.8791, \mathrm{~V}(\mathrm{Y})=0.0394, \mathrm{E}(\mathrm{Z})=0.9956, \mathrm{~V}(\mathrm{Z})=0.0053$

The correlation coefficient of the corresponding indicators is obtained from Pearson correlation coefficient:

$$
\begin{aligned}
& r(\mathrm{~A} 1, \mathrm{~A} 2)=r(\mathrm{~A} 2, \mathrm{~A} 1)=0.9546, \mathrm{r}(\mathrm{A} 1, \mathrm{~A} 3)=\mathrm{r}(\mathrm{A} 3, \mathrm{~A} 1)=0.0334, \\
& \mathrm{r}(\mathrm{A} 2, \mathrm{~A} 3)=\mathrm{r}(\mathrm{A} 3, \mathrm{~A} 2)=0.0352, \mathrm{r}(\mathrm{A} 1, \mathrm{~A} 1)=\mathrm{r}(\mathrm{A} 2, \mathrm{~A} 2)=\mathrm{r}(\mathrm{A} 3, \mathrm{~A} 3)=1
\end{aligned}
$$

We know that qualified rate of the user terminal voltage and load rate will be related strongly, then other indicators are not related each other. Finally, two indicators will be filtered by the correlation for modeling analysis .

Indicators of fusion. According to the two indicators of user voltage qualified rate and load rate, take each set of data values of DSS, and establish the probability boxes. As is shown, Figure 3 shows two indicators' weighted fusion algorithm under the condition of no correlation each other. Figure 4 shows, the correlation factor of the Lucas correlation model embedded weighted fusion improves fusion algorithm under the strongly related conditions.

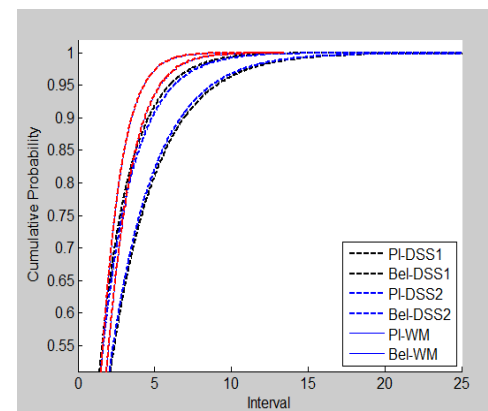

Figure 3: Independence

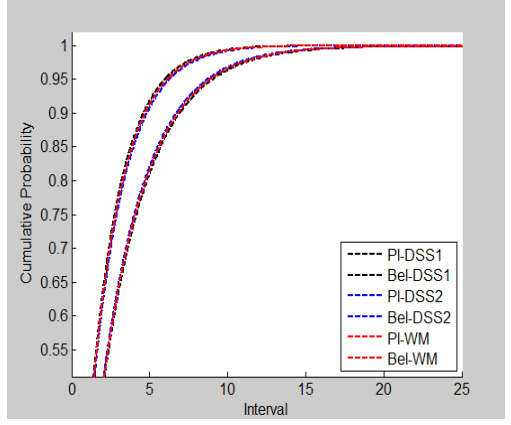

Figure 4: Positive

\section{Analysis of experimental results}

The results of the Figure 3 and 4 are numerical quantitatively analyzed, by using the method of defining integral area.Definite the fused overlap factor to $\delta$, so:

$$
\delta=\frac{2 B 3}{B 1+B 2} \times 100 \%
$$

To illustrate different correlations under different conditions, we take different parameters to produce positive correlation. take DSS of each set, establish the probability boxes, and then compare with strongly correlation. It is concluded, overlap factor contrast value as shown in Table 2:

Table 1. Relevance of the Results under Different Conditions

\begin{tabular}{ccccc}
\hline $\begin{array}{c}\text { Experiment } \\
\text { Type }\end{array}$ & \multicolumn{2}{c}{ Positive correlation } & \multicolumn{2}{c}{ Strong positive correlation } \\
\hline Algorithm & Independent & Perfect & Independent & Perfect \\
Overlap factor & $76 \%$ & $100 \%$ & $52.2 \%$ & $90.6 \%$ \\
\hline
\end{tabular}

From Figure 3,4 and Table 2, the fusion result is more reasonable under the condition of positively strong correlation and positively all correlation than the condition of independent irrelevance. The result after fusion under positively strong correlation should be almost the same with the before, 
but the Figure 3 obviously dissatisfy. In this paper, the correlation of the other, also made the corresponding modeling results are by default of fusion algorithm has improved significantly, In this paper, the correlation of the other, also making the corresponding modeling of others, the result has a significant improvement.

\section{Conclusion}

This paper proved the different result when use different fusion method of the probability box. Aim at the pre-indexes of power grid planning, do the weighted fusion algorithm under independent unrelated conditions and compared with weighted fusion algorithm of Lucas correlation model based on strong positive correlation and full-related. The results shows that the latter is more effective in improving the fusion algorithm. Therefore, use the method of probability box do the fusion of indexes and correlation analysis before fusion algorithm will help improve the reasonable of probability box modeling.

\section{Acknowledgements}

This research is sponsored by the National Science Foundation of China No. 51467007, and the Application Basic Research Plan in Yunnan Province of China No. 2013FZ020.The corresponding author of this paper is Ding Jiaman.

\section{References}

[1] GORENSTIN B G, CAMPODONICO N M, COSTA J P. Power system expansion planning under uncertainty .IEEE Trans on Power Systems.1993,8(4): 129-136.

[2] Nie Hongzhan, NIE Song, Qiao Yi, et al. Comprehensive decision making of alternative transmission network planning based on principal component analysis $[\mathrm{J}]$. Power System Technology, 2010, 34(6): 134-138.

[3] Zhou Fang, Wang Pengbo, Han Liyang. Multiple source knowledge fusion processing algorithms. Journal of Beijing University of Aeronautics and Astronautics. 2013,01(39):109-114

[4] SCOTT FERSON, VLADIK KREINOVICH, LEV GINZBURG, et al. Constructing Probability Boxes and Dempster-Shafer Structures[M]. Sandia National Laboratories, 2003:143-180.

[5] Ding Jiaman, Du Yi, Wang Qingxin, Jia Lianyin etc. Fault Diagnosis of Power Transformer based on Probability-box Theory, Advances in Intelligent Systems Research, 2014,101:419-422 .

[6] Li Xinran, Li Peiqiang, Zhu Xiangyou,et. Evaluation Construction Size of District High Voltage Distribution Networks. Automation of Electric Power Systems. 2007,31(4):46-50.

[7] Cheng Hua,Du Siwei,Xu Cuihua,et al. A DS-Based Multi-index Fusion of Information Fusion Algorithm. Journal of East China University of Science and Technology(Natural Science Edition) .2011,08(37):483-486

[8] SCOTT FERSON, ROGER B.NELSEN, JANOS HAJAGOS, et al. Dependence in probabilistic modeling, Dempster-Shafer theory, and probability bounds analysis[M]. Sandia National Laboratories, 2004:121-191. 